\title{
Machine vision methods for estimation of size distribution of aggregate transported on conveyor belts
}

\author{
Michal Kontny \\ Silesian University of Technology, Gliwice, Poland \\ E-mail: michal.kontny@polsl.pl
}

Received 18 September 2017; accepted 19 September 2017

DOI https://doi.org/10.21595/vp.2017.19151

Check for updates

\begin{abstract}
The article describes a set of methods for estimation of volume and size distribution of aggregate transported on conveyor belts. The objective was accomplished by means of machine vision and image processing techniques. Three dimensional shape of the rock pile was acquired using the method of laser triangulation. Image processing algorithm was developed in order to outline separate grains and thus allowing to infer on the size distribution of the bulk material transported. Further calculations performed on the raw data allowed to assess its volume.
\end{abstract}

Keywords: rock size distribution, laser triangulation, conveyor belt, machine vision, image processing.

\section{Introduction}

Belt conveyors are a widely used form of transporting bulk goods on short and medium distances in many branches of industry. In spite of substantial initial investment cost, they provide economical means of moving large quantities of bulk materials. Belt conveyor transport is not only ubiquitous but also in large extent indispensable in the heavy industry including mining of rock, ore and coal.

Efficient operation of those industries requires precise monitoring of the flow of material during extraction, processing and shipping. The author's initial intention was to concentrate his efforts in the area of coal mining but solutions developed can be implemented in other industries dealing with aggregate.

The coal can be described by large number of chemical and physical parameters determining its quality and technical properties. The area of volume and size distribution measurement was identified as promising ground for development of real time measuring system. The automation of measurement of the fore-mentioned parameters (especially size distribution) could not only cut the cost of manual sampling but also, providing real-time information, allow better control of the plant processes [4].

This paper presents a portion of a wider research that resulted in integration of a laboratory test rig adapted for testing of data acquisition methods and data processing algorithms suitable for accomplishing the goal of automated measurement of above mentioned parameters.

The accuracy of the system was validated both during preliminary laboratory trials and also during field test carried out in a coal mine located in the Upper Silesia region in Poland.

In this paper the author will briefly describe the principles behind the data acquisition system and then present the methods used for extraction of useful data describing material properties.

\section{Measurement method}

The recent years have brought a wide application of commercial machine vision systems in many industries. The use of machine vision provides economical solutions in the area of process and quality control. The conventional approach encompassing the acquisition, processing and analysis of images of the investigated object has been successfully used in many industrial applications. Some researchers have also tried to use standard vision techniques to characterize bulk material transported on conveyor belts. This approach, in terms of size distribution assessment, has many flaws and so far, has been mostly unsuccessful. 
The bulk material transported on the conveyor belt forms a pile consisting of irregularly shaped grains overlapping each other. Both of these factors impair the ability to precisely detect the boundaries between the grains, thus substantially reducing the ability to produce accurate size distribution of the lot.

Different approach has been proposed with the introduction of three-dimensional vision techniques. Obtaining an accurate surface profile not only allows precise delineation of adjacent grains but also brings additional information concerning height of the pile, hence allowing the calculation of the volume.

Obtaining the surface profile has been made available by the implementation of the laser triangulation method.
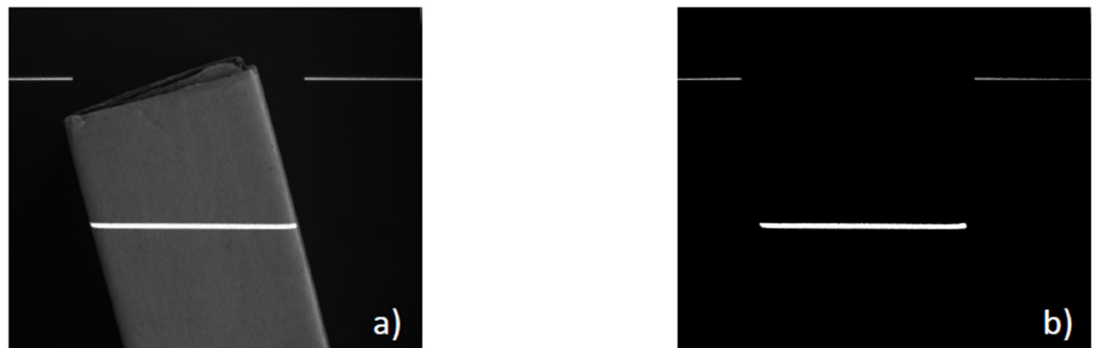

Fig. 1. Grayscale image of the cuboid cardboard box with the laser profile (a), laser line profile after thresholding operation (b) [1]

The line-shaped laser source pointing at the conveyor belt at an angle produces a distinct line profile captured by the camera mounted above the belt surface. Using geometric relationships and assuming a chosen perspective model one can obtain the information about the actual height of the illuminated region [2]. A series of consequent measurements can provide enough information to reconstruct the height profile of an object. In the case considered, the conveyor provides the inline motion, whereas the speed camera captures a series of images.

Fig. 1 shows the principle of laser triangulation method. The change of the shape of the laser line profile captures the height of the examined object. The further the laser line deviates from the reference point (flat surface of the conveyor belt), the higher the elevation of the particular region of the object. The laser line profile is extracted from the grayscale image using thresholding.

The calculated surface profile is represented in the form of height map, that is a matrix, where an 8 bit value representing the height of the corresponding point is assigned to each of its elements. The height map can be interpreted as an image and undergo further processing. In the image representation, the brighter regions represent areas that are higher, whereas darker spots represents lower parts of the object.

The inclination of the laser source, necessary for the height measurement, results in the small areas overshadowed by the bigger rocks. In order to calculate the volume of the material, it is necessary to obtain a continuous surface profile. For this purpose, a custom interpolation method has been developed to fill in the "black voids" visible in the picture Fig. 2.

\section{Image processing algorithms}

Prototyping and testing of the image acquisition, processing and analysis system were carried out using LabVIEW environment.

The raw height map Fig. 2 was assembled using the height information calculated from a series of thresholded laser profile images. The light intensity reflects the actual height of the surface.

A custom interpolation method was used to provide continuous surface profile. The regions corresponding to the flat surface of the belt conveyor were assigned a value of zero height, the rest of the image was scaled to the range of 0-255 (conforming with 8 bit format). A combination 
of basic filtering and morphological operations was used in order to smooth out the surface, having in consideration the objective of retaining distinct boundaries between the grains Fig. 3.

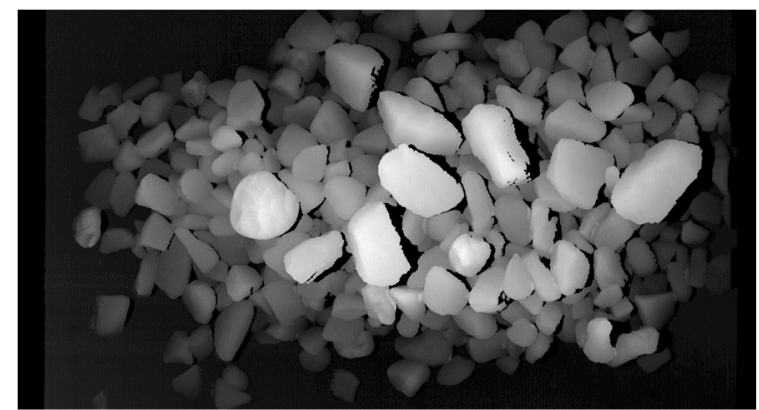

Fig. 2. Height map obtained from raw laser line images [1]

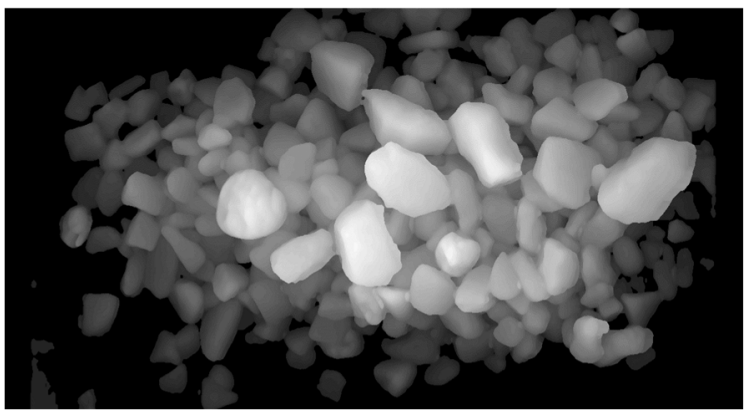

Fig. 3. Height map after applying interpolation algorithm followed by further image processing operations [1]

Edge detection was a crucial stage of the image processing algorithm. The goal of this operation was to bring out the boundaries separating adjacent grains. The algorithm and the parameters of the constituent operations had to be carefully engineered in order to find the right balance between not being able to detect existing boundaries and detecting too many artificial boundaries (caused e.g. by irregular shape of the grains). Laplace edge detection filter was used, followed by a series of morphological operations Fig. 4.

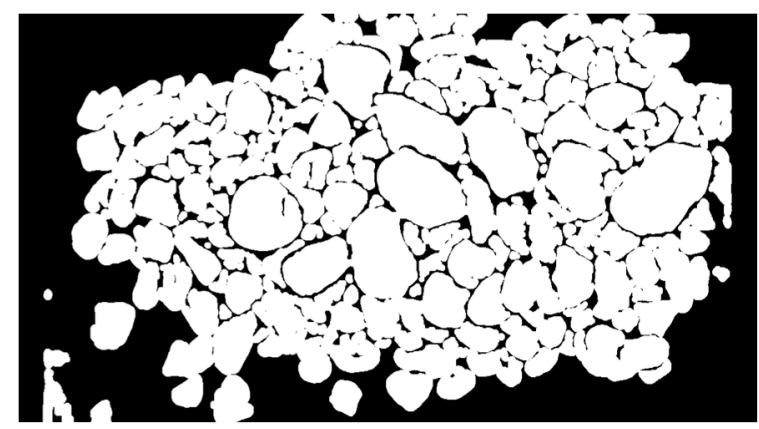

Fig. 4. Edge detection using Laplace filter followed by a set of morphological operations [1]

Further stages involved application of Danielsson filter Fig. 5 followed by Watershed Segmentation and other intermediate image processing operations.

Combination of Danielsson transform and Watershed Segmentation enables effective segmentation of the image, especially in cases where the exact position of boundaries between objects is difficult to identify. The Fig. 6 shows the final segmentation of the image. 


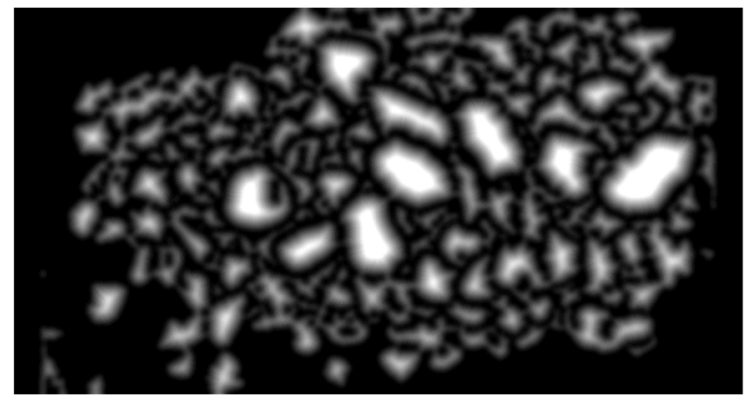

Fig. 5. Image after Danielsson transform followed by simple arithmetic operations [1]

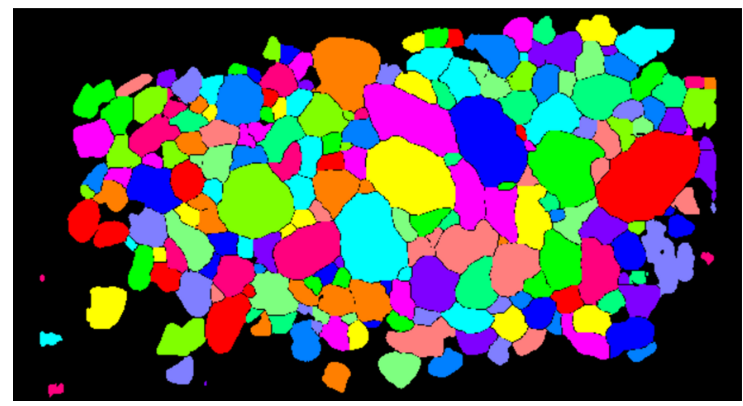

Fig. 6. Image after segmentation [1]

\section{Results}

The image subjected to segmentation enables the extraction of selected features describing shape and size of detected grains. In the aggregate industry, the size distribution of the bulk material is usually assessed in the course of sieve analysis, where the shape and size of consequent sieves determines the allocation of the grain to the specific size class.

In the course of the research it was decided that for the purpose of preliminary validation of the segmentation method it was sufficient to use the area characteristic.

The Fig. 8 shows the histogram representing the size distribution of the tested sample, characterized by the area (measured in pixels).

A further assumption was made that surface of the rock pile transported on conveyor belt, in terms of size distribution, is a good representation of the whole batch. Practical experience shows that due to the vibration generated along the path of the conveyor, the larger fractions tend to travel towards the surface of the pile, whereas the smaller rocks tend to fill in the voids and concentrate in the lower and middle layers of the pile. The matter of using the surface profile of the aggregate as the representation of the cross-section of the pile was contemplated by Mathew J. Thurley [5] et al.

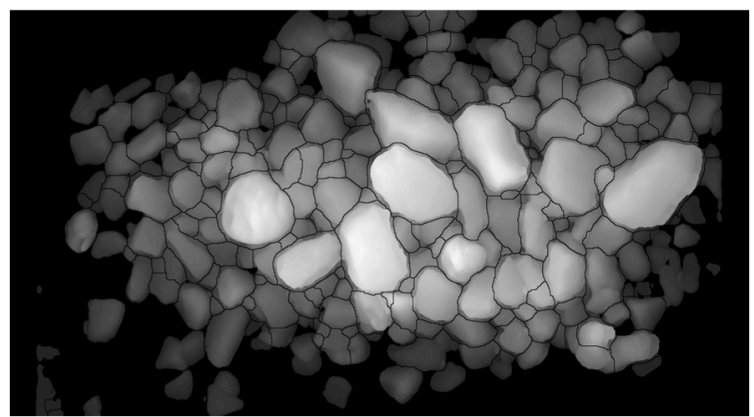

Fig. 7. Height map with delineated grain boundaries [1] 
This assumption holds if the measurement is carried out in a place where the mentioned reposition has not yet occurred.

In order to achieve desired accuracy of the result, it is also necessary to identify grains overlapped by their adjacent neighbours [3]. Using three-dimensional profile of the surface makes this detection possible, but during this research this method was not implemented.

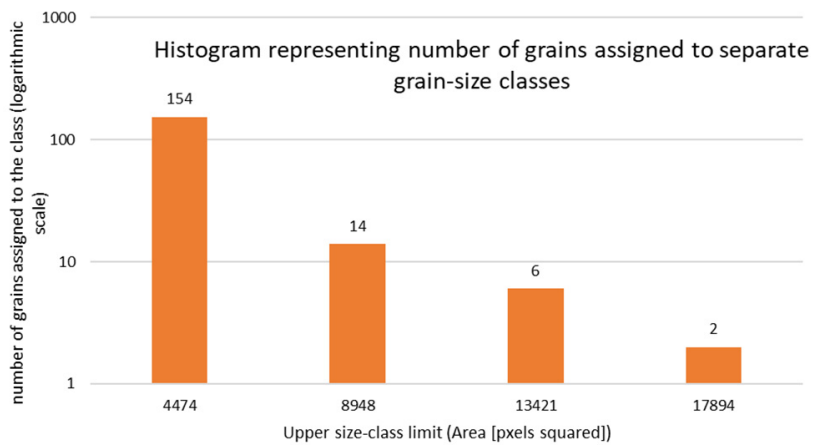

Fig. 8. Histogram representing number of detected grains assigned to separate size classes [1]

The accuracy of the segmentation showed in Fig. 7 has been subjectively assessed as satisfactory. The boundaries of grains, in spite of large diversity of the sample (grain size), are deemed to have been detected accurately. The histogram presented in Fig. 8 represents the number of detected grains assigned to four different size classes.

\section{Conclusions}

Both the proposed 3D data acquisition approach and following image processing and analysis methods were found to be successful for the task of determining the size distribution of the aggregate transported on the conveyor belts. The measurements and analysis were carried out automatically, without the need for the manual intervention of the operator.

In the course of the research, the image processing methodology has proved to be flexible enough to cope with different rock types and grain sizes. In all of the cases the sequence of the image processing operations remained unchanged. In some cases, where distinctly different samples were introduced, some of parameters governing image processing operations had to be fine-tuned manually.

This leaves space for further research involving introduction of self-adaptive mechanisms allowing full automation of the system in order to cope with a wide variety of materials without human supervision.

\section{References}

[1] Kontny M. Application of Data Processing and Analysis Techniques for the Inspection of Mine Output Transported on Conveyor Belts. Master's Thesis, Gliwice, 2016.

[2] Kontny M. Review of selected methods for control of quantity and quality of the coal mine output transported on conveyor belts. Interim Paper, Gliwice, 2016.

[3] Thurley M. J., Ng K. C. Identification and sizing of the entirely visible rocks from a 3D surface data segmentation of laboratory rock piles. Journal of Vibroengineering, Computer Vision and Image Understanding, Vol. 111, 2008, p. 170-178.

[4] Thurley M. Fragmentation Size Measurement Using 3D Surface Imaging. CRC Press, 2009, p. 229-237.

[5] Thurley M. Automated, On-Line, Calibration-Free, Particle Size Measurement Using 3D Profile Data, CRC Press/Balkema, 2012, p. 229-237. 